\title{
Conservative averaging as an approximate method for solution of some direct and inverse heat transfer problems
}

\author{
A. Buikis \\ Institute of Mathematics, Latvian Academy of Sciences and \\ University of Latvia, Latvia
}

\begin{abstract}
The conservative averaging method was developed as an approximate analytical and/or numerical method for solving partial differential equation or its system with piece-wise constant (continuous) coefficients. The usage of this approximate method for separate relatively thin sub-domain or/and for subdomain with al large heat conduction coefficient leads to a reduction of domain in which the solution must be found. To apply this method for all sub-domains of layered media, a special type of spline was constructed: the integral averaged values interpolating parabolic spline. The usage of this spline allows diminishing the dimensions of initial problem per one. It is important that in all cases the original problem with discontinuous coefficients from $R^{n+1}$ transforms to problem with continuous coefficients in $R^{n}$. A method of conservative averaging for ill-posed inverse problems in some cases allows transforming them to wellposed inverse problems.
\end{abstract}

Keywords: heat transfer, piecewise constant (continuous) coefficients, conservative averaging, non-classical conditions, integral spline, mesh (dimension) reduction, direct problem, inverse problem.

\section{Introduction}

By modeling practically interesting processes, e.g. heat transfer processes in non-homogeneous media, very often we need to consider the situation, when the medium has an organized structure, i.e. it is not fully chaotic. For example it often has a layered structure. In addition some of these layers are relatively thin in comparison with adjacent layers and have strongly different physical properties. 
Mathematically speaking such a situation can be described by a partial differential equation (or its system) with piecewise constant/continuous coefficients, i.e. the domain in which the solution is defined, consists of several sub-domains. In each sub-domain the solution of the partial differential equation can be considered in the classical sense: the solution has continuous all highest partial derivations into the sub-domain. But it is not true on the contact surfaces $S$ of adjacent layers: on these surfaces special additional conditions, which in the literature are often called conjugation or junction conditions, have been formulated: $[T]=0,\left[k \frac{\partial T}{\partial n}\right]=0$. Here $[T]=\left.T\right|_{S^{+}}-\left.T\right|_{S^{-}}$is the difference of one-sided limit values (jump) of function $T$ on surface $S$. In the case of non-ideal contact one of the conditions is the continuity of heat flux (energy conservation). The second junction or conjugation condition is usually written without detailed deduction. E.g., [1] mentions "temperature has discontinuity when passing through the boundary of non-ideal contact, with the height of the step being proportional to the heat flow, i.e.

$$
[T]=\frac{k}{\alpha} \frac{\partial T}{\partial n},(x, y, z) \in S,
$$

where the coefficient of contact heat transfer $\alpha$ is associated with the contact conditions". Similarly, in [1] the so-called "concentrated heat capacity" condition relation on the surface $S$ is written as follows

$$
\left[k \frac{\partial T}{\partial n}\right]=c_{s} \rho \frac{\partial T}{\partial t} .
$$

Additionally, it commented that: " $c_{s}$ is the lumped heat capacity of the contact".

In this paper we will show how these and other conditions and their generalizations can be obtained by our original method of conservative averaging (CAM) [2, 3]. This approach allows us to eliminate some separate sub-domains and reduce partial differential equations for these sub-domains to boundary conditions. This means that we reduce the definitions domain of problem for its analytical or numerical solution [4]. This means we can consider this approach using the mesh reduction method. To apply CAM procedure for several layers, it was necessary to construct a special type of spline: the integral averaged values interpolating parabolic spline [5]. In [6] such an approximation for convectionconduction heat transfer in a layered system was demonstrated. For the approximation of boundary layers we introduced rational spline [7] and in [8] we showed its effectiveness. It is important that in all the cases the original problem with discontinuous coefficients transforms from problem a in $R^{n+1}$ to problem a in $R^{n}$ with continuous coefficients. This method for ill-posed inverse problems in some cases allows transforming them to well-posed inverse problems, e.g. $[9,10]$. 


\section{The conservative averaging method for separate boundary sub-domain}

Let us assume that in suitable Cartesian coordinates $x \in R, y \in R^{n}$ the domain of definition $\bar{D} \subset R^{n+1}$ of the solution is represented as consisting of two sub-domains: $\bar{D}=\bar{G} \cup \overline{G_{0}}$. Here the finite or infinite sub-domain $G$ has form $G=\{(x, y) \mid x \in(0, \Delta(y)), y \in \Gamma(x)\}$ and the second sub-domain $G_{0}=\left\{(x, y) \mid x \in(-\delta, 0), y \in \Gamma_{0}\right\}$ is a cylinder of finite height $\delta$. The base of the cylinder $\Gamma_{0} \in R^{n}$ is the bounded/unbounded domain in $R^{n}$ under the condition $\Gamma_{0} \subseteq \Gamma(0)$. The differential equation in $G_{0}$ has the form:

$$
\frac{\partial}{\partial x}\left(k_{0} \frac{\partial U_{0}}{\partial x}\right)+L_{0}\left(U_{0}\right)=-F_{0}(x, y),
$$

where $L_{0}$ is the linear differential operator with respect to the argument $y$ and coefficient $k_{0}=k_{0}(y)$. The differential equation in $G$ has a similar form:

$$
\frac{\partial}{\partial x}\left(k \frac{\partial U}{\partial x}\right)+L(U)=-F(x, y),
$$

but the operator $L$ with respect to the vector argument $y$ now in the general case can be non-linear and the heat conduction coefficient is $k=k(x, y)$. It should be mentioned that one of the $y$ vector components may be time $t$, thus the equations (3) and (4) allow describing both the steady-state and transient processes. Let us denote by $\Gamma_{0}^{+}$the part of the hyper-plane $x=0$ : $\Gamma_{0}^{+}=\bar{G} \cap \overline{G_{0}}$. On this surface the conjugations conditions must be fulfilled:

$$
\begin{gathered}
\left.U_{0}\right|_{x=-0}=\left.U\right|_{x=+0}, \\
\left.k_{0} \frac{\partial U_{0}}{\partial x}\right|_{x=-0}=\left.k \frac{\partial U}{\partial x}\right|_{x=+0}
\end{gathered}
$$

On the second base $\Gamma_{0}^{-}=\left\{x=-\delta, y \in \Gamma_{0}\right\}$ of the cylinder, a typical boundary conditions for the heat transfer processes is given (we will specify it later). On the other hand we will not specify the conditions of the remaining parts of both sub-domains, because their form is not substantial for the description of the method.

We assume that the original problem (3)-(6) with all the necessary boundary or/and initial conditions have a unique and stable solution. In particular it should be emphasized that under the solution of this problem we imply a solution in a 
slightly revised classical sense: 1) it is continuous in closure of definition domain $\bar{D} ; 2$ ) the solution has all necessary continuous highest derivations in open sub-domains $G_{0}$ and $G ; 3$ ) the first derivative with respect to argument $x$ of the solution has bounded one-sided limit values which fulfil the second junction condition (6) on surface $\left.\Gamma_{0}^{+} ; 4\right)$ it fulfils all additional conditions on boundary of definition domain.

We start the description of the CAM by introducing the integral averaged value of the function $U_{0}(x, y)$ of the solution of the problem (3)-(6) on $\bar{G}_{0}$ :

$$
u_{0}(y)=\delta^{-1} \int_{-\delta}^{0} U_{0}(x, y) d x
$$

The integration of the differential equation (3) over the interval $x \in(-\delta, 0)$ and the utilization of second junction condition give us the basic relation

$$
\left.k \frac{\partial u}{\partial x}\right|_{x=+0}-\left.k_{0} \frac{\partial U_{0}}{\partial x}\right|_{x=-\delta}+\delta L_{0}\left(u_{0}\right)=-\delta f_{0}(y) .
$$

Firstly, we denote the solution on sub-domain $\bar{G}$ by $u(x, y)$ instead of the function $U(x, y)$ because the solution of the new statement of the problem in general will differ from initial solution. Secondly, we denote by $f_{0}(y)$ according to (7) the averaged value of source function $F_{0}(x, y)$. A further transformation of the basic relation (8) depends on the type of boundary conditions on $\Gamma_{0}^{-}$. We will consider the boundary condition of second type (other types of boundary condition were considered in [2, 3]):

$$
-\left.k_{0} \frac{\partial U_{0}}{\partial x}\right|_{x=-\delta}=\varphi^{0}(y)
$$

Assuming the linear approximation for solution $U_{0}(x, y)$ for each fixed $y$ (nevertheless it may be different for various $y$ !) we easily find the representation for $u_{0}(y)$ by means of the junction condition (5) and condition (9):

$$
u_{0}(y)=u(0, y)+\frac{\delta}{2} \frac{\varphi^{0}(y)}{k_{0}(y)}
$$

It remains to then insert this expression in (8), to use boundary condition (9) and we have obtained the non-classical boundary condition on boundary $\Gamma_{0}^{+}$: 


$$
k \frac{\partial u}{\partial x}+\delta L_{0}(u)=-\left[\varphi^{0}(y)+\delta f_{0}(y)+\frac{\delta^{2}}{2} L_{0}\left(\frac{\varphi^{0}}{k_{0}}\right)\right]
$$

for the solution $u(x, y)$ of the equation (4) on the reduced domain $G$ :

$$
\frac{\partial}{\partial x}\left(k \frac{\partial u}{\partial x}\right)+L(u)=-F(x, y) .
$$

Of course all additional conditions of the original problem on the remaining part of boundary of domain $G$ must be added to main differential equation (11) and new boundary condition (10) on the surface $\Gamma_{0}^{+}$. When this new problem is solved it is easy to obtain a posteriori error estimation $\Delta U_{0}(x, y)$ for the linearly approximate (relatively to $x$ ) solution $U_{0}(x, y)$ in following form:

$$
\begin{gathered}
\left|\Delta U_{0}(x, y)\right| \leq \frac{|x|}{k_{0}} \times \\
\times\left|\left[k \frac{\partial u(0, y)}{\partial x}+\varphi^{0}(y)\right]+\frac{\delta}{2}\left[L_{0}\left(u_{0}\right)+\frac{\delta}{2} L_{0}\left(\frac{\varphi^{0}}{k_{0}}\right)+\delta^{2} f_{0}(y)\right]\right|
\end{gathered}
$$

We can increase the order of approximation for solution $U_{0}(x, y)$ up to two by means of both junction conditions and condition (9). We get the representation:

$$
U_{0}(x, y)=u(0, y)+\frac{k}{k_{0}}\left(1+\frac{x}{2 \delta}\right) x \frac{\partial u(0, y)}{\partial x}+\frac{x^{2} \varphi^{0}(y)}{2 \delta k_{0}} .
$$

We obtain from (12) following expression for the averaged integral value of the function $U_{0}(x, y)$ :

$$
u_{0}(y)=u(0, y)+\frac{\delta}{3 k_{0}}\left[\frac{\varphi^{0}(y)}{2}-k \frac{\partial u(0, y)}{\partial x}\right] .
$$

This means that the new boundary condition on surface $\Gamma_{0}^{+}$consists of two equations. One of them is a consequence of equation (8):

$$
\left.k \frac{\partial u}{\partial x}\right|_{x=+0}+\delta L_{0}\left(u_{0}\right)=-\left[\varphi^{0}(y)+\delta f_{0}(y)\right],
$$

the second one is expression (13). These two equations allow finding two unknown functions $u_{0}(y)$ and $u(0, y)$ on the boundary $\Gamma_{0}^{+}$. So the new problem consists of the main differential equation (11) together with a system of two non-standard boundary conditions (13), (14). The definition domain for the 
function $u(x, y)$ is sub-domain $\bar{G}$; the function $u_{0}(y)$ is given only on the surface $\bar{\Gamma}_{0}^{+}$.

So the CAM in this situation can be interpreted as the mesh reduction method. We finish this part of paper with some remarks. Quite often after the application of CAM with piecewise constant coefficients it is possible to solve the problem analytically. Our experience concerning the numerical methods for other problems with non-classical boundary conditions is as follows. Firstly, we have solved a large number of important practical problems (mostly by the method of finite difference). Secondly, in practice and in theory the stability criterion for classical boundary condition (9) is more stiff than for the non-classical boundary condition (10) (or for the system (13) and (14)). Unfortunately still we haven't succeeded in proving the solvability of problems with non-classical additional conditions in general (for general operators $L_{0}$ and $L$ ).

\section{The conservative averaging method for separate inner sub-domain (layer)}

Now we will consider the definition domain $D$ which consists of three sub-domains: $\bar{D}=\bar{G} \cup \overline{G_{0}} \cup \overline{G_{1}}$, where finite or infinite sub-domain $G_{1}$ has the form $G_{1}=\left\{(x, y) \mid x \in\left(-\Delta_{1}(y),-\delta\right), y \in \Gamma_{1}(x)\right\}$. We add to equations (3) and (4) in sub-domain $G_{1}$ equation

$$
\frac{\partial}{\partial x}\left(k_{1} \frac{\partial U_{1}}{\partial x}\right)+L_{1}\left(U_{1}\right)=-F_{1}(x, y)
$$

and to conditions (5) and (6) add further junction conditions on surface $\Gamma_{0}^{-}$:

$$
\left.U_{1}\right|_{x=-\delta-0}=\left.U_{0}\right|_{x=-\delta+0},\left.k_{1} \frac{\partial U_{1}}{\partial x}\right|_{x=-\delta-0}=\left.k_{0} \frac{\partial U_{0}}{\partial x}\right|_{x=-\delta+0} .
$$

The basic relation for this problem looks as follows:

$$
\left.k \frac{\partial u}{\partial x}\right|_{x=+0}-\left.k_{0} \frac{\partial u_{0}}{\partial x}\right|_{x=-\delta+0}+\delta L_{0}\left(u_{0}\right)=-\delta f_{0}(y) .
$$

To exclude thin interlayer - cylinder $G_{0}-$ and to obtain the new non-standard junction conditions on surface $\Gamma_{0}^{+}$we shift the sub-domain $G_{1}$ to the right: $x \mapsto x+\delta$ and additionally assume the linear approximation for solution $U_{0}(x, y)$ for each fixed $y$. Then from (6) and (16) immediately follows the first junction condition on $\Gamma_{0}^{+}$: 


$$
\left.k_{1} \frac{\partial u_{1}}{\partial x}\right|_{x=-0}=\left.k \frac{\partial u}{\partial x}\right|_{x=+0}
$$

The second junction condition on $\Gamma_{0}^{+}$follows from (17) by taking into consideration linearity of function $U_{0}(x, y)$ :

$$
\left.k \frac{\partial u}{\partial x}\right|_{x=+0}-\frac{k_{0}}{\delta}[u]+\frac{\delta}{2} L_{0}\left(u+u_{1}\right)=-\delta f_{0}(y) .
$$

If we neglect in (19) both the operator $L_{0}$ and source term then this condition reduces to the non-ideal contact condition. In addition, we have explicated the expression for the coefficient of contact heat transfer $\alpha$ in (1) through physical and geometrical properties of the interlayer: $\alpha=\frac{k_{0}}{\delta}$. The "concentrated heat capacity" condition (2) follows from the basic relation (17) with operator $L_{0}=c_{0} \rho_{0} \frac{\partial}{\partial t}, f_{0} \equiv 0$ and equality (18). The explicit expression for the lumped heat capacity $c_{s}$ derives from (17) and is as $c_{s}=c_{0} \delta$.

\section{The coefficient inverse one-dimensional problem for two- layer system}

One of most popular experimental methods for thermal physical properties of homogeneous media (solids, fluids and gases) with low electrical conductivity is the transient hot strip (THS) method developed by Gustafsson [11]. Mathematically this method was formulated as coefficient inverse heat equation with constant coefficients for two-dimensional semi-bounded zone. In our publications $[9,10]$ we generalize this ill-posed problem for two-layers, solving it by use of Green's function and reducing it to a system of two transcendent equations. Nevertheless, the numerical solution of system of transcendental equations is an ill-posed problem. It would be important to offer some well-posed method for finding approximate values for coefficients as initial data for the iteration process. In this section we propose such an approach based on conservative averaging. The one-dimensional model for the THS method can be formulated as follows:

$$
\begin{aligned}
& c_{0} \frac{\partial U_{0}}{\partial t}=k_{0} \frac{\partial^{2} U_{0}}{\partial x^{2}}+f_{0} \delta(0), 0<x<H_{0}, 0<t, \\
& c_{1} \frac{\partial U_{1}}{\partial t}=k_{1} \frac{\partial^{2} U_{1}}{\partial x^{2}}, H_{0}<x<H_{0}+H_{1}=H, 0<t
\end{aligned}
$$

with homogeneous second type boundary conditions 
with conjugation conditions

$$
\left.k_{0} \frac{\partial U_{0}}{\partial x}\right|_{x=0}=0,\left.\quad k_{1} \frac{\partial U_{1}}{\partial x}\right|_{x=H}=0
$$

$$
\left.U_{0}\right|_{x=H_{0}-0}=\left.U_{1}\right|_{x=H_{0}+0},\left.k_{0} \frac{\partial U_{0}}{\partial x}\right|_{x=H_{0}-0}=\left.k_{1} \frac{\partial U_{1}}{\partial x}\right|_{x=H_{0}+0}
$$

and with homogeneous initial conditions

$$
\left.U_{0}\right|_{t=0}=0,\left.U_{1}\right|_{t=0}=0 .
$$

Additional information is given:

$$
\left.U_{0}\right|_{x=0}=T(t)
$$

in the form $T\left(t_{k}\right)=T_{k}, t_{k}=k \Delta t, k=\overline{0, N}$ with $N>>1$. The aim of the solution of the inverse problem is to find the unknown constants $c_{0}, c_{1}$ and functions $U_{0}(x, t)$ and $U_{1}(x, t)$.

Let us introduce the integral averaged values of functions $U_{0}(x, t)$ and $U_{1}(x, t)$ :

$$
u_{0}(t)=\frac{1}{H_{0}} \int_{0}^{H_{0}} U_{0}(x, t) d x, \quad u_{1}(t)=\frac{1}{H_{1}} \int_{H_{0}}^{H} U_{1}(x, t) d x .
$$

Further, we will approximate both unknown functions $U_{0}(x, t), U_{1}(x, t)$ according to argument $x$ with expressions, which fulfil the boundary and conjugation conditions and equalities (23). Finally we have:

$$
\begin{aligned}
& U_{0}(x, t)=u_{0}(t)-\frac{e G_{0}}{2\left(G_{0}+G_{1}\right)}\left(e^{\frac{x}{H_{0}}}+e^{-\frac{x}{H_{0}}}+e^{-1}-e\right) v(t), \\
& U_{1}(x, t)=u_{1}(t)+\frac{e G_{1}}{2\left(G_{0}+G_{1}\right)}\left(e^{\frac{x-H}{H_{1}}}+e^{\frac{H-x}{H_{1}}}+e^{-1}-e\right) v(t),
\end{aligned}
$$

where $v(t)=u_{0}(t)-u_{1}(t), G_{i}=H_{i} k_{i}^{-1}, i=0,1$.

Now we integrate the main equations (20) and (21):

$$
c_{0} \frac{d u_{o}}{d t}=\left.G_{o}^{-1} \frac{\partial U_{0}}{\partial x}\right|_{x=H_{0}-0}+f_{0}, \quad c_{1} \frac{d u_{1}}{d t}=\left.G_{1}^{-1} \frac{\partial U_{1}}{\partial x}\right|_{x=H_{0}+0} .
$$

Finally we receive a system of two ordinary differential equations:

$$
\beta_{0} \frac{d u_{0}}{d t}=-\alpha v(t)+f_{0} H_{0}, \quad \beta_{1} \frac{d u_{1}}{d t}=\alpha v(t),
$$

with homogeneous initial conditions $u_{0}(t)=u_{1}(t)=0$. 
Here $\alpha=\frac{e^{2}-1}{2\left(G_{0}+G_{1}\right)}$ and $\beta_{i}=c_{i} H_{i}, i=0,1$. The solution for $v(t)$ is:

$$
v(t)=f_{0} H_{0}\left(\beta_{0} \gamma\right)^{-1}\left(1-e^{-\gamma t}\right), \gamma=\alpha \delta, \delta=\beta_{0}^{-1}+\beta_{1}^{-1} \text {. }
$$

And the solution for $u_{0}(t)$ :

$$
u_{0}(t)=f_{0} H_{0} \beta_{0}^{-1}\left[\left(\beta_{0} \gamma \delta\right)^{-1}\left(1-e^{-\gamma t}\right)+\left(1-\left(\beta_{0} \gamma\right)^{-1}\right) t\right] .
$$

We have from additional information (22):

$$
T_{k}=u_{0}\left(t_{k}\right)+\alpha_{0} v\left(t_{k}\right), \alpha_{0}=2^{-1}\left(e^{2}-2 e-1\right) G_{0}\left(G_{0}+G_{1}\right)^{-1} .
$$

The first finite difference may be written as follows:

$$
\Delta T_{k}=f_{0} H_{0}\left(\beta_{0} \gamma\right)^{-1}\left[\left(\alpha_{0}+\left(\beta_{0} \delta\right)^{-1}\right)\left(1-e^{-\gamma \Delta t}\right) e^{-\gamma t_{k-1}}+\left(\gamma-\left(\beta_{0}\right)^{-1}\right) \Delta t\right]
$$

and we have similar expression for the second finite difference:

$$
\Delta^{2} T_{k}=-f_{0} H_{0}\left(\beta_{0} \gamma\right)^{-1}\left[\left(\alpha_{0}+\left(\beta_{0} \gamma\right)^{-1}\right)\left(1-e^{-\gamma \Delta t}\right)^{2} e^{-\gamma t_{k-1}}\right] .
$$

Finally, we obtain expression for the sum of the unknown coefficients:

$$
\gamma=\frac{1}{\Delta t} \ln \frac{\Delta^{2} T_{k-1}}{\Delta^{2} T_{k}}
$$

Evidently we have found the unique solution of the inverse problem, but it is easy to see that solution (26) strongly depends on the errors of the measurements and in this sense we have unstable algorithm. But we can propose the modification of this algorithm by summing up the sub-sequences of the measurement data.

\section{References}

[1] Samarskii, A.A., Vabishchevich, P.N., Computational Heat Transfer. Vol.1, Mathematical Modelling. John Wiley\&Sons Ltd., 1995.

[2] Buikis, A. Aufgabenstellung und Loesung einer Klasse von Problemen der mathematischen Physik mit nichtklassischen Zusatzbedingungen. Rostock. Math. Kolloq., 1984, 25, pp. 53-62. (In German.)

[3] Buikis, A., The change of formulation of problems of mathematical physics with discontinuous coefficients in compound domains. The Electronic Modelling, 1986, Vol. 8, No. 6, pp. 81-83. (In Russian.)

[4] Buikis, A., Buike, M., Guseinov, Sh., Analytical two-dimensional solutions for heat transfer in system with rectangular fin. Advanced Computational Methods in Heat Transfer VIII. WIT Press, 2004, pp.35 44.

[5] Buikis, A., Interpolation of integral averaged values of piecewise smooth function by means of parabolic spline. Latvian Mathematical Yearbook, 1985, No. 29, pp. 194-197. (In Russian.) 
[6] Buikis, A., Ulanova, N., Analytically-numerical method for temperature fields in multilayered system. Advanced Computational Methods in Heat Transfer VI. WIT Press, 2000, pp. 445-453.

[7] Buikis, A., Rational integral values interpolating spline and its properties. Latvian Mathematical Yearbook, 1988, No.32, pp. 173-182. (In Russian.)

[8] Buikis, A., Jegorovs, J., Application of the conservative averaging for the filtration problem with large velocity. Mathematical Modelling and Analysis, 2001, Vol.6, Nr.2, pp. 251-261.

[9] Guseinov, Sh., Buikis, A., Inverse heat transport problems for coefficients in two-layer domains and methods for their solution. Mathematical Modelling and Analysis, 2002, Vol. 7, N 2, Vilnius, "Technika", pp. $217-$ 228.

[10] Buikis, A., Guseinov, Sh., Conservative averaging method for solutions of inverse problems of mathematical physics. Progress in Industrial Mathematics at ECMI 2002. A. Buikis, R. Ciegis, A. D. Fitt (Eds.), Springer, 2004, p. 241-246.

[11] Gustafsson, S. L., Karawacki, E., Khan, M. N., Transient hot-strip method for simultaneously measuring thermal conductivity and diffusivity of solids and fluids. J. Phys. D: Appl. Phys., 1979, Vol. 12, pp. 1411-1421. 